\title{
Personen- und Aufgabenmerkmale beim komplexen Problemlösen
}

\author{
Regina Vollmeyer und Joachim Funke
}

\begin{abstract}
Zusammenfassung. In diesem Überblick werden Ergebnisse zum komplexen Problemlösen dargestefl, die anhand von linearen Systemen gewonnen wurden. Zuerst wird definiet, was lineare Sysieme sind. Die Ergebnisse werden getrennt nach Personen- oder Aufgabenmerkmalen aufgeführt. Als Personenmerkmale wurden die Variablen Motivation, Intelligenz und Strategien untersuchk, Aufgabenmerkmale waren Semantische Einkleidung, Zielspezifitit, Hypothesentesten und Wissenserwert. Zur Integration der Ergebnisse in ein theoretisches Modell wird auf das Zwei-Räume-Modell von Klahr und Dunbar (1988) verwiesen.

Schlüsselwöner. Komplexes Problemlösen, Lineare Systeme, Zwei-Räume-Modell
\end{abstract}

\section{Person and task effects within complex problem solving}

\begin{abstract}
This overview presents results on complex problem solving, in which tasks are linear systems. At the beginning, we define what a linear system is. Then we separated the resulhs those due 10 person or lask chancteristics. Person characteristics considered are motivation, inselligence and strategies; task characteristics are semantic embedding, gaal specificity, hypothesis testing and knowledge acquisition. To inkegrale the results we apply the deal-space model by Klahr and Dunbar (1988).

Key words: complex problem solving, dual-space model, linear systerns
\end{abstract}

Die Mitte der siebziger Jahre von Dietrich Dörner im - deutschsprachigen Raum initiierte Forschung zum „Komplexen Problemlösen" hat verschiedene Ausformungen erhalten. Während sich die sogenannte „Bamberger Schule" mit der Konstruktion semantisch komplexer Umwelten ohne besondere Rücksicht auf deren formale Struktur beschäftigte, hat sich die "Bonner Schule“ vor allem den formal einheitlich konstruierten Systemen ohne große Rücksicht auf deren Semantik gewidmet. Dieser Beitrag befaßt sich mit komplexen Problemstellungen, die unter Zuhilfenahme des Formalismus der linearen Gleichungssysteme konstruiert wurden.

Wie sieht ein solches System für einen Problemlöser konkret aus? Typischerweise wird den Problemlösern als Einführung erklärt, daB sie in einem System Variablen (sog. Eingangsvariablen) verändern können, die dann andere $V$ ariablen beeinflussen (sog. Ausgangsvariablen). Die Struktur für ein derartiges einfaches lineares System ist in Abbildung I dargestellt.

Die Vorbereitungen zu diesem Artikel wurden von der Deutschen Forschungsgemeinschaft gefördent durch ein Stipendium an Regina Vollmeyer (Vo $514 / 1$ ) und durch Sachmittelbeihilfen an Regina Vollmeyer und Falko Rheinberg (Vo 514/5) sowie an Joachim Funke (Fu 173/7). Für Kommentare zu einer Erstfassung bedanken wir uns bei Johannes Engelkamp und Burkhard Müller.

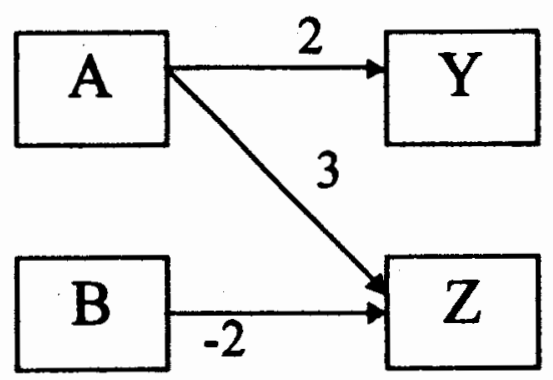

Abbildung l. Struktur eines einfachen linearen Systems mit zwei Eingangsvariablen A und B sowie zwei Ausgangsvariablen $Y$ und $Z$, die gemäß der angegebenen $K a n-$ ten und Gewichte verknüpft sind.

Hier sind $A$ und $B$ die Eingangsvariablen, die auf die Ausgangsvariablen $\mathrm{Y}$ und $\mathrm{Z}$ wirken. Die Zahlen an den Pfeilen geben Gewichte an, mit denen die jeweilige Eingangsvariable auf die Ausgangsvariable wirkt. Formal ist das System durch zwei Gleichungen beschreibbar, nämlich:

$$
\begin{aligned}
& Y_{1+1}=2 \cdot A_{1} \\
& Z_{1+1}=3 \cdot A_{1}-2 \cdot B_{1}
\end{aligned}
$$


Dabei gibt der tiefer gestellte Index jeder Variablen den jeweiligen Zeitpunkt ( $t$ bzw, $t+l$ ) des Systems an, der in diskreten Stufen getaktet wird. Aus Gleichung (1) ergibt sich also, daß der Wert der Variable Y zum Zeitpunkt $t+l$ sich ergibt aus dem Wert der Variable A zum Zeitpunkt $t$, multipliziert mit 2 .

Meistens wird so ein Syslem am Computer dargeboten, wobei durch die Darbietung erkennbar ist, welche Variablen manipuliert werden können. Nicht erkennbar ist, ob und welche Eingangsvariable welche Ausgangsvariable/n beeinfluBt. In manchen Systemen wirken sogar manche Ausgangsvariablen auf andere Ausgangsvariablen (sog. Nebenwirkungen) oder eine Ausgangsvariable verändert sich konstant, auch wenn das System nicht manipuliert wird (sog. Eigendynamik). Durch Verwendung solcher Systemmerkmale können beliebig komplexe lineare Gleichungssysteme konstruiert werden.

Nach dieser ersten Beschreibung, was wir unter linearen Systemen verstehen, und bevor wir mit unserem kurzen Überblick über bereits vorliegende empirische Befunde beginnen, sind einige Abgrenzungen und Definitionen nötig.

Probleme versus Aufgaben. Dörner (1987, S. 10) unterscheidet zwischen Problemen und Aufgaben, wobei Aufgaben geistige Anforderungen sind, für deren Bewältigung Methoden bekannt sind. Während bei Aufgaben reproduktives Denken eingesetzt wird, muB bei Problemen etwas Neues entstehen. Dieser Unterscheidung wollen wir aus zwei Gründen nicht nachgehen und nur von Systemen reden: Der erste Grund ist eher pragmatisch, nämlich in der angloamerikanischen Literatur wird diese Unterscheidung nicht getroffen. Dort gibt es nur tasks. Der zweite Grund ist, daß die Unterscheidung darauf hinausläuft, daß vor dem Einsatz eines Systems für die betreffende Stichprobe untersucht werden müBte, ob die Probanden Methoden zur Bearbeitung kennen oder nicht. $\mathrm{Da}$ die linearen Systeme längere Zeit zur Lösung beanspruchen, sollte bei erfolgreicher Bearbeitung aus dem Problem eine Aufgabe werden. Der von uns hier präferierte Begriff „System“ vermeidet derartige Unklarheiten. In der Kybernetik sind Systeme Maschinen oder Organismen, die Eingangs- und Ausgangsvariablen haben, welche miteinander vernetzt sind. Für den vorliegenden Überblick wollen wir unter dem Begriff des Systems auch solche Aufgabenstellungen zulassen, die jeweils nur eine Ausgangsvariable haben, wie z. B. den Wert einer Aktie bestimmen (Earley, Connolly \& Ekegren, 1989). Auch solche Aufgaben, die nur aus einer Gleichung bestehen, sind Gegenstand dieses Überblicks und werden im folgenden als Systeme bezeichnet.

Linear versus nicht-linear. Um den Überblick einzuschränken, werden wir nur Ergebnisse von Systemen mit linearen Gleichungen berücksichtigen. Ausgeschlossen werden daher Aufgaben zum Hypothesentesten, wie z. B. die Teile eines elektrischen Regelkreises (Schauble, Glaser, Raghavan \& Reiner, 1992), die Funktion einer Taste (Klahr \& Dunbar, 1988) oder Regeln zur Joghurtproduktion (Schoppek, 1997) herauszufinden. Auch eher realistische Systeme, die z. B. das Landen von Flugzeugen
(Kanfer \& Ackerman, 1989) oder Umschiffen von Minen (Gordon \& Subramanian, 1997) simulieren, werden ausgeschlossen, obwohl eine Theorie des Problemlösens, die die Identifikation und Kontrolle linearer Systeme erklärt, grundsätzlich auch für diesen Aufgabentyp gültig sein sollte.

Die Einschränkung auf Systeme, denen lineare Strukturgleichungen zugrunde liegen, mag auf den ersten Blick verwundern. Bei genauerem Hinsehen wird allerdings deutlich, daß natürlich mit linearen Systemen sehr wohl nichtlineare Entwicklungen über die Zejt hinweg modelliert werden können. Die enorme Potenz linearer Gleichungssysteme erkennt man u.a. daran, daB fast das gesamte methodische Arsenal der Psychologie auf linearer Algebra aufbaut.

\section{Überblick über verschiedene Forschungsbereiche}

Wenn wir schon den Überblick über Ergebnisse zum Problemlösen durch den verwendeten Aufgabentyp einschränken, so wollen wir für die Darstellung der Befunde diese pragmatische Sichtweise aufgeben. Die Ergebnisse mit diesem Aufgabentyp werden daher nach Themen unterteilt berichtet. Dabei kann untersucht werden, wie gut Probanden die Zusammenhänge in dem System identifizieren können (Wissenserwerb) und ob sie es so kontrollieren können, daß ein vorgegebener Zielzustand erreicht wird (Wissensanwendung).

\section{Motivation, Selbstwirksamkeit}

Alltagspsychologisch würde man annehmen, daB Motivation das Kontrollieren eines linearen Systems beeinfluBt, und zwar sollten Hochmotivierte mehr Wissen über die Struktur des Systems erwerben und/oder genauer den Zielzustand erreichen. Wie jedoch läßt sich Motivation erfassen? Möglich ist, Motivation wie ein Persönlichkeitsmerkmal vor der Aufgabenbearbeitung zu erfassen und mit Leistung zu korrelieren. Dies hat Süß (1996) getan, indem er Selbstwirksamkeitserwartung (Schwarzer \& Jerusalem, 1981) sowie Leistungsorientierung und Beanspruchung (Fahrenberg, Hampel \& Selg, 1984) gemessen hat, bevor er das lineare System Tomaten bearbeiten lieB. Die Korrelationen gehen zwar in die erwartete Richtung, aber nur einige haben eine geringe bis mittlere Effektstärke mit verschiedenen Leistungsmaßen.

Bandura und Kollegen (Bandura \& Wood, 1989; Wood \& Bandura, 1989; Wood, Bandura \& Bailey, 1990) betonen dagegen, daß nicht die generalisierte Selbstwirksamkeit das relevante $\mathrm{MaB}$ ist, sondern vielmehr die während der Aufgabenbearbeitung entstehende Selbstwirksamkeit. Daher bitten sie ihre Probanden zu mehreren Meßzeitpunkten einzuschätzen, wie sicher sie sich sind, bestimmte Werte zu erreichen. Die Aufgabe der Probanden bestand darin, als Manager einer Möbelfabrik fünf Arbeiter Unterproduktionen zuzuweisen, um eine 
gute Produktion der Firma zu erreichen. Da in den Experimenten mehrere Meßzeitpunkle vorlagen, konnte in Pfadanalysen gezeigt werden, daß eine aktuelle hohe Selbstwirksamkeit dazu führt, daB analytischere Strategien gewählt werden. Diese analytischen Strategien und die hohe Selbstwirksamkeit direkı führten zu einer guten Leistung.

Die Motivation während der Aufgabenbearbeitung kann jedoch davon abhängen, ob jemand Erfolg oder Mißerfolg erlebt und dadurch seine Fähigkeit einschätzen kann. Vollmeyer und Rheinberg (1998) gehen in ihrem kognitiv-motivationalen Prozeßmodell daher davon aus, daß die Motivation (vier Komponenten: Erfolgszuversichtlichkeit, Mißerfolgsbefürchtung, Interesse, Herausforderung) nach dem Lesen der Aufgabenbeschreibung, aber noch vor der Aufgabenbearbeitung die relevante Motivation ist. Ähnlich wie bei Bandura und Kollegen wird betont, daß die Motivation auch indirekt über Strategien und Motivation während der Aufgabenbearbeitung auf den Lemproze $\beta$ wirken kann. Erste Studien dazu mit der linearen Aufgabe Biology Lab bestätigen das Modell (Vollmeyer, Rollett \& Rheinberg, 1997). Insgesamt kann also von einem EinfluB von Motivation auf das Problemlösen mit linearen Aufgaben ausgegangen werden.

Obwohl den Themen Motivation und Metakognition unterschiedliche Theorieansätze zugrunde liegen, so werden sie zum Teil ähnlich definiert und operationalisiert (Weinert, 1984). Metakognition beim Lemen eines komplexen Systems wurde in einer Studie von Vollmeyer und Rheinberg (in press) mit Hilfe des Lauten Denkens untersucht.

\section{Intelligenz}

Neben der Motivation könnte als Personenmerkmal auch die Intelligenz ein Prädiktor für die Leistung beim $\mathrm{Be}$ arbeiten linearer Aufgaben sein. Da es bereits einen Überblick über ältere Studien gibt (Beckmann \& Guthke, 1995; SüB, 1996) und H.-M. Süß in diesem Themenheft eine aktuelle Übersicht gibt, wird hier auf eine Darstellung verzichtet.

\section{Strategien}

Tschirgi (1980) identifizierte verschiedene Strategien, um den Einfluß von mehreren Eingangsvariablen auf eine oder mehrere Ausgangsvariablen zu untersuchen. Will man z. B. herausfinden, was einen Kuchen besser macht (mehr Butter, weniger Eier), so ist es eine sehr systematische/analytische Strategie, nur eine Variable zu verändern, und dagegen eine sehr unsystematische, alle Variablen auf einmal zu verändern. Solche Strategien lassen sich besonders bei linearen Aufgaben festmachen (Bandura \& Wood, 1989; PreuBler, 1998; Putz-Osterloh, 1993; Vollmeyer, Bums \& Holyoak, 1996; Wood \& Bandura, 1989; Wood, Bandura \& Bailey, 1990). In allen Studien zeigte sich der Befund, daB systematischere Strategien zu einer besseren Leistung führen.

\section{Semantische Einkleidung}

Während die ersten drei Themen (Motivation, Intelligenz und Strategien) Personenvariablen sind, handelt es sich bei den nachfolgenden Themen um Aufgabenmerkmale. In Funkes (1995) Überblick über komplexes Problemlösen wird bereits die Studie von Beckmann (1994) erwähnt, bei der das isomorphe lineare System entweder als Szenario Kirschbaum oder als Maschine vorgegeben wurde. Während bei der abstrakten Maschine Wissen über die Struktur des Systems erworben wurde, lemten die Probanden bei der Darbietung des strukturgleichen Systems Kirschbaum wenig über die Struktur, da sie die Komplexität der Struktur nicht reduzieren konnten. Leider liegen zu wenige kontrollierte Experimente zu dieser Thematik vor, so daB eine Theorie und Replikationen nötig sind, um vorherzusagen, wie die Semantik auf den Wissenserwerb und die Kontrolle bei linearen Aufgaben wirkt.

\section{Zielspezifität}

Ein in der Arbeits- und Organisationspsychologie häufig untersuchtes Aufgabenmerkmal ist die Zielspezifität (s. eine Metaanalyse von Tubbs, 1986). Eine typische Manipulation dieser Variable ist, daß einer Gruppe ein spezifisches Ziel gegeben wird (,Erreiche den Wert 20!"), einer anderen ein unspezifisches („Gib Dein Bestes!"). Übertragen auf lineare Systeme bedeutet dies, daB in der Bedingung mit spezifischem Ziel die Zielwerte, auf die das System gebracht werden soll, bekannt sind, während das unspezifische Ziel lautet: „Versuche herauszufinden, wie die Eingangs- mit den Ausgangsvariablen verknüpft sind!“" In den meisten Studien, die in älteren Überblicken (Funke, 1991, 1995) referiert wurden, ist nur die Bedingung mit spezifischem Ziel realisiert. Erst in neueren Experimenten mit linearen Systemen wird diese Unterscheidung als relevant für den Wissenserwerb erachtet.

Eine erste Serie von drei Experimenten liegt von Earley, Connolly und Ekegren (1989) vor. Hier sollten Probanden den Aktienpreis für insgesamt hundert Firmen vorhersagen, der aus immer der gleichen linearen Formel berechnet werden kann. Die Guppe mit spezifischem Ziel soll maximal eine Abweichung von nur $\$ 10$ von dem tatsächlichen Wert haben, während die Gruppe mit dem unspezifischen Ziel ihr Bestes geben sollte. Diese Variation der Versuchsbedingungen bewirkte, daß die Gruppe mit spezifischem Ziel schlechtere Preisschätzungen geben konnte als die Gruppe mit dem unspezifischen Ziel.

Mit dem weiter oben erwähnten System, in dem die Probanden Manager einer Möbelfabrik sind, manipulierten Cervone, Jiwani und Wood (1991) Zielspezifität. Einer Gruppe wurde ein konkretes Ziel vorgegeben (mittlere oder hohe Schwierigkeit), während die andere Gruppe keinen Zielwert erhielt, sondern einfach ihr Bestes geben sollte. Diese Manipulation hatte keinen Effekt auf Leistung, sie moderierte allerdings die selbstregulatorischen Prozesse: für Probanden mit einem spezi- 
fischen Ziel waren motivationale Variablen wie Selbstwirksamkeit, Selbstbewertung und Höhe der selbstgesetzten Ziele gute Prädiktoren, für Probanden mit einem unspezifischen Ziel waren sie nicht effektiv.

Mit einem typischeren linearen System als dem soeben genannten, dem erwähnten Biology Lab, variierten Vollmeyer, Burns und Holyoak (1996) die Zielspezifität wie folgt: In einer Explorationsphase kannte die Gruppe mit spezifischem Ziel (SZ) bereits die Zielwerte, die in einer Anwendungsphase zu erreichen waren. In der Anwendungsphase erhielt dann auch die Gruppe mit unspezifischem Ziel (UZ) die Zielwerte. Es folgte eine Transferphase, in der das gleiche System auf neue Zielwerte gebracht werden sollte. In der Explorationsphase lernte die UZ-Gruppe mehr über die Verbindungen zwischen den Eingangs- und Ausgangsvariablen als die SZ-Gruppe. In der Anwendungsphase gab es keinen Unterschied darin, wie genau die Zielwerte erreicht wurden. Das heißt, die UZ-Gruppe konnte ihr gutes Wissen erfolgreich einsetzen, die SZ-Gruppe hat dagegen gelernt, mit weniger Wissen über die Struktur des Systems die Zielwerte zu erreichen. In der Transferphase erreichte die UZ-Gruppe die neuen Zielwerte besser als die SZGruppe, da sie transferierbares Wissen erworben hatte.

Eine ähnliche Aufgabe verwendeten Geddes und Stevenson (1997), nämlich die Interaktionsaufgabe von Berry und Broadbent (1984), in der die Probanden mit der Computerperson Clegg kommunizierten und sie deren Zustand durch Eingaben, wie rude, cool, usw. verändern konnten. In diesem Experiment gab es drei verschiedene Gruppen, die Gruppe mit spezifischem Ziel (SZ), die Clegg in einen bestimmten Zustand versetzen sollte, die Gruppe mit unspezifischem Ziel (UZ), die das Muster in Cleggs Reaktionen herausfinden sollte, sowie eine Gruppe mit beiden Zielen. Die Aufgabenbearbeitung war in jeweils 30 Explorations- und Transferdurchgänge (neues Ziel für alle Gruppen) aufgeteilt. Zusätzlich wurde das Wissen über das System erhoben. Auch hier fand sich das Ergebnis, daB die UZ-Gruppe das meiste Wissen über das System hatte und in den Transferdurchgängen die meisten Durchgänge richtig machte.

Zusammenfassend läßt sich sagen, $\mathrm{daB}$ es bei linearen Systemen zentral darauf ankommt, wie das Ziel formuliert wird. Liegt ein spezifisches Ziel vor, versuchen die Probanden, das Ziel zu erreichen, ohne über die dem System zugrundeliegenden Regeln nachzudenken. Daher haben sie ein geringeres Wissen als Probanden, die ein unspezifisches Ziel hatten. Die SZ-Gruppe kann dieses ungenügende Wissen auch nicht auf neue Ziele übertragen.

\section{Hypothesentesten}

Eng verbunden mit der Frage nach den verwendeten Strategien, der semantischen Einkleidung und der Zielspezifität ist die, ob Hypothesen getestet werden: Eine systematische Strategie sollte ein Indikator dafür sein, daß der Proband eine Hypothese formuliert hat (z. B. die Ein- gangsvariable $\mathrm{A}$ wirkt auf Ausgangsvariable $\mathrm{X}$ ); die semantische Einkleidung liefert Hinweise darauf, wie Eingangs- und Ausgangsvariablen zusammenhängen könnten (z. B. Licht und Kirschen im Szenario Kirschbaum von Beckmann, 1994); bei einem unspezifischen Ziel sucht der Proband nach derartigen Systemzusammenhängen. Wenn Probanden also Hypothesen formulieren, anstatt Ziele zu erreichen oder unsystematisch Variablen zu verändern, sollte mehr Wissen erworben und folglich das System besser kontrolliert werden. Dies wurde von Vollmeyer und Burns (1996) überprüft. In zwei Experimenten gaben sie einer Gruppe eine Hypothese, einer anderen Gruppe nicht. Im ersten Experiment war die Hypothese über eine sehr leichte Verbindung in dem System, so daB die Probanden nicht zusätzlich Informationen erhielten, im zweiten Experiment war die Hypothese sogar falsch. Sogar mit falscher Hypothese erwarb die Gruppe mit Hypothese mehr Wissen über das System, was zeigt, daB es für das Lernen wichtig ist, bei linearen Aufgaben explizit Hypothesen zu formulieren.

\section{Wissenserwerb}

Das am häufigsten untersuchte Thema ist, ob und wie beim Kontrollieren linearer Systeme Wissen erworben wird. Das Thema läßt sich in drei spezifische Fragestellungen unterteilen:

1. Wie modelliert man den Wissenserwerb am besten?

2. Was wird durch Beobachten ohne eigenes Eingreifen gelernt?

\section{Wird implizites oder explizites Wissen erworben?}

Modellierung des Wissenserwerbs. Bevor man eine Computersimulation des Wissenserwerbs vornehmen kann, ist es nötig, ein theoretisches Modell des Lernprozesses zu haben. Beckmann (1994) legt seinem Modell den Ansatz multinomialer Modellierung (Riefer \& Batchelder, 1988) zugrunde. Mit Hilfe dieses Modells überprüft Beckmann, warum bei zwei isomorphen Systemen (Maschine, Kirschbaum) einmal Wissen erworben wurde, das andere Mal nicht. Es gelingt ihm dabei zu zeigen, daß der Proze $B$ des Wissenserwerbs nicht der gleiche war.

Die wohl am weitesten verbreitete Lerntheorie ist die ACT-R-Theorie von Anderson (1993; Anderson \& Lebiere, 1998). Ein erster Versuch, den Wissenserwerb und das Kontrollieren eines linearen Systems mit diesem Modell zu beschreiben, wurde von Schoppek (1998) unternommen. Auch bei Wallach (1998) findet sich ein derartiger Formalisierungsversuch für ein dynamisches System.

Beobachten vs. Eingreifen. Bereits 1988 ließen Funke und Müller eine Gruppe das Sinus-System kontrollieren, während eine zweite Gruppe sie dabei beobachtete. Obwohl einem Probanden-Paar, bestehend aus einem Eingreifer und einem Beobachter, die gleichen Daten vorlagen, korrelierten die Leistungen des Paares nicht. Die Probanden, die in das System eingreifen konnten, lernten 
mehr darüber, welche Eingangs- und Ausgangsvariablen zusammengehören. Dagegen wußten die Beobachter eher, wie stark der Einfluß 2wischen den Variablen ist.

Berry (1991) untersuchte ebenfalls die Leistung von Eingreifern und Beobachtern und fand, da $B$ Eingreifer bessere Resultate erbrachten. Sie interpretierte dies derart, $\mathrm{da} B$ das aktive Handeln sich förderlich auf die Leistung auswirkt.

Komplizierter sind die Annahmen von Stevenson, Geddes, Sumner und Travis (1998). Sie gehen von einer Interaktion von Zielspezifität (s. o.) und Beobachten aus. In einem Experiment zeigten sie, daß, wenn Beobachter ein unspezifisches Ziel hatten (nämlich das System zu verstehen), sie genauso gut waren wie Probanden, die in das System eingreifen konnten. Dies Experiment verdeutlicht wieder, wie wichtig es ist zu wissen, weiches Ziel die Bearbeiter der linearen Systeme verfolgen.

Implizites vs. explizites Wissen. Seit der Studie von Berry und Broadbent (1984), die keinen Zusammenhang zwischen Wissen und Steuerleistung fanden, wird untersucht, ob implizites, d.h. nicht verbalisierbares Wissen beim Kontrollieren linearer Systeme erworben wird (für einen Überblick: Buchner, 1992; Seger, 1994; SüB, 1996). Im folgenden werden wir nur auf Studien eingehen, die neueren Datums sind.

SüB (1996) untersuchte den Zusammenhang von Wissen und Steuerungsleistung bei dem System Tomaten, wobei Wissen so erfaßt wurde, daB die Probanden während des Explorierens die Verbindungen zwischen den Eingangs- und Ausgangsvariablen in ein Diagramm einzeichneten. Zwischen dem Vorwissen und dem während des Explorierens gemessenen Wissen gab es keinen Zusammenhang, dagegen korrelierte letzteres hoch mit der Steuerungsleistung.

PreuBler (1996) argumentiert, daß die Methode, das Wissen zu erfassen, indem man Diagramme vorgibt, das Explorieren eines linearen Systems verändert. Diese Methode bewirke, daB explizit gelernt wird. Sie schlägt vor, für explizites Wissen eine Paar-Aufgabe, und für implizites Wissen eine lexikalische Entscheidungsaufgabe einzusetzen. An ihrem System Linas erzielte sie das Ergebnis, daß das explizite Wissen kein Prädiktor für das Zielerreichen war. Dagegen hatten diejenigen Probanden, die in der lexikal ischen Entscheidungsaufgabe gut waren, einen Vorteil bei den leichten Variablen des Systems.

In einer Nachfolgeuntersuchung untersuchte Preußler (1998), ob tatsächlich eine explizite Wissensvermittlung zu einer besseren Transferleistung führt. Dazu wurde der Experimentalgruppe während einer Übungsphase das Strukturwissen vermittelt (Experiment 1) oder die KonIrollgruppe bekam zusätzlich ein spezifisches Ziel (Zielspezifität, s.o.) in der Übungsphase (Experiment 2). In beiden Experimenten lernte die Experimentalgruppe in der Lernphase mehr über die Zusammenhänge im System und konnte dieses Wissen besser auf eine ähnliche Aufgabe übertragen.

Eine weitere Dissoziation von Identifikation und Kontrolle eines linearen Systems fand Preußler (1997) bei
Probanden, die neben den Variablen, die sie zur Zielerreichung benötigten, noch zugleich irrelevante $V$ ariablen erhielten. Wurde das Linas-System dagegen auf die relevanten Variablen reduzien, zeigte sich ein Zusammenhang zwischen dem Wissen über die Struktur des Systems und der Steuerleistung.

Um auf die vielen hier nicht aufgeführten Ergebnisse zu dieser Fragestellung zu generalisieren, wäre es nötig, eine Metaanalyse zu berechnen. Darauf haben wir hier verzichtet. Unser Eindruck ist, daß bei einfacheren Systemen (ohne Zeitverzögerung, ohne Nebenwirkungen), die noch dazu in eine Explorations- und Anwendungsphase unterteilt sind, das Wissen über die Zusammenhänge im System die Steuerleistung vorhersagt.

\section{Integration in ein Rahmenmodell}

Nachdem wesentliche Ergebnisse, die mit linearen Systemen gewonnen wurden, dargestellt sind, stellt sich die Frage, ob sie in ein Rahmenmodell integriert werden können und dadurch eine theoretische Erklärung bekommen. Die Forschung mit linearen Systemen begann unter dem Begriff Komplexes Problemlösen, folglich müßte eine Theorie des Problemlösens die Ergebnisse erklären. VanLehn (1989) stellte in seinem Überblicksartikel zum Problemlösen allerdings fest, daB seit dem klassischen Modell von Newell und Simon (1972) kein neues Modell aufgestellt wurde. Newell und Simons Modell beschreibt, wie Personen Probleme lösen. Problemlösen wird als Suche in einem Problemraum gesehen. Der Problemraum eines Problems besteht aus drei Komponenten: 1. dem Anfangszustand, 2. den Operatoren, mit denen die $\mathrm{Zu}$ stände transformiert werden können, und 3. einem Test, ob der Zielzustand erreicht ist. Darauf baut der. Ansatz des Zwei-Räume-Modells von Simon und Lea (1974) auf, die den Problemraum in einen Regelraum und einen Instanzenraum unterteilen. Im Regelraum sind alle möglichen Regeln einer Aufgabe enthalten, im Instanzenraum die möglichen Zustände. Am Beispiel linearer Systeme sind die Regeln alle möglichen Verbindungen und Gewichte zwischen den Eingangs- und Ausgangsvariablen. Die Zustände sind alle möglichen Werte, die die Ausgangsvariablen einnehmen können. Die Operatoren sind die Werte, die man den Eingangsvariablen zuweisen kann.

Klahr und Dunbar (1988) griffen den Ansatz des Zwei-Räume-Modells auf. Sie entwickelten das SDDSModell (,Scientific Discovery as Dual Search"), um wissenschaftliche Entdeckungen zu erklären. In diesem Modell ist der Instanzenraum ähnlich dem Experimenteraum und der Regelraum ist ähnlich dem Hypothesenraum. Im Hypothesenraum werden Hypothesen über $z$. B. Verbindungen zwischen Eingangs- und Ausgangsvariablen generiert, modifiziert und verworfen. Im Experimenteraum werden dagegen Experimente geplant, wie die generierten Hypothesen überprüft werden können oder wie die Operatoren anzuwenden sind. Dazu müssen beide Problemräume (wie schon bei Simon \& Lea, 1974) interagieren: Aktivitäten im Hypothesenraum lösen Operatio- 
nen im Experimenteraum aus. Es gibt auch die umgekehrte Einflußrichtung: Wenn keine Hypothese über Beobachtungen zu dem System aufgestellt wird (Suche im Hypothesenraum), ist es nämlich möglich, Operatoren anzuwenden (Suche im Experimenteraum). Durch Beobachtung der Ergebnisse dieser Experimente können dann Hypothesen abgeleitet werden.

Mit dem Zwei-Räume-Modell lassen sich die Ergebnisse zu den Strategien, der semantischen Einkleidung, der Zielspezifität, dem Hypothesentesten und dem Wissenserwerb erklären. In vielen Untersuchungen wurde nicht zwischen einer Explorations- und Anwendungsphase unterschieden, d.h. die Probanden kannten von Anfang an die Zielwerte oder den Zielzustand ihres Systems (spezifisches Ziel). Damit läßt sich diese Aufgabe auch so lösen, daß Personen mit einer Mittel-ZielAnalyse versuchen, das Ziel anzusteuern (Suche im Instanzenraum), ohne Hypothesen zu formulieren. Sie erwerben dadurch kein Wissen über das System, lernen aber, wie das Ziel zu erreichen ist (implizites Wissen). So haben u.a. Geddes und Stevenson (1997) die Dissoziation von Wissen und Zielerreichen erklärt. Wenn explizites Wissen erworben wird, dann liegt Hypothesengenerierung und -testung vor (Suche im Regelraum). Die Suche im Regelraum kann dadurch gefördert werden, daß eine systematische Strategie verwendet wird (Strategie) und keine Zielwerte vorgegeben werden (Zielspezifität). Eine semantische Einkleidung sowie das Vorgeben einer Hypothese haben zur Folge, daß Hypothesen getestet werden und somit die Suche im Regelraum gefördert wird.

Mit Hilfe des Zwei-Räume-Modells lassen sich die Ergebnisse interpretieren und es wird einsichtig, warum bei manchen Aufgaben etwas gelernt wurde und bei anderen nicht. Dennoch gibt es Befunde, die nun wiederum eine Erweiterung des Modells nötig machen. Ein solcher Befund ist, daB manchmal ein spezifisches Ziel zu besseren Leistungen führt und zwar dann, wenn die Probanden ein nicht passendes Modell der Aufgabe haben (Burns \& Vollmeyer, 1996). Neben einer Erweiterung des Modells gibt es auch Alternativerklärungen für die Befunde, wie Zielspezifität das Lemen beeinflussen kann (Nhouyvanisvong \& Koedinger, 1998). Besonders dieses Thema scheint eine gute Schnittstelle für Theorieentwicklung und Empirie zu sein.

\section{Fazit}

Aufgrund ihrer klaren formalen Struktur eignen sich lineare Systeme ausgezeichnet für die systematische Manipulation von Aufgabencharakteristika. Die hierdurch wie auch durch andere (die Personencharakteristika betreffende) experimentelle Manipulationen nachgewiesenen Effekte erlauben den Aufbau eines Wissensfundus zu diesem Bereich komplexen Problemlösens, der durch einfache theoretische Annahmen über die Vorgänge im Problemraum modelliert werden kann. Auch wenn diesen Untersuchungen gelegentlich der "Flair“" komplexer Problemlöseforschung zu fehlen scheint, wie er von GroßSzenarien wie Lohhausen (Dörner, Kreuzig, Reither \&
Stäudel, 1983) seinerzeit ausging: Wichtig erscheint uns, daß zum einen mit diesem Ansatz die wesentlichen Charakteristika des Phänomenbereichs (Komplexität, Vernetztheit, Intransparenz, Polytelie, Dynamik) abgebildet werden können, zum anderen gerade durch die Überschaubarkeit der Szenarien eine theoriegeleitete Modellierung ablaufender Problemlöseprozesse erleichtert wird.

\section{Literatur}

Anderson, J. R. (1993). Rules of the mind. Hillsdale, NJ: Entbaum.

Anderson, J. R. \& Lebiere, C. (Eds.). (1998). The atomic components of thought. Mahwah, NJ: Erlbaum.

Bandura, A. \& Wood, R. E. (1989). Effect of perceived controllability and performance standards on self-regulation of complex decision making. Journal of Personalin and Social Psychology, 56, 805-814.

Beckmann, J. (1994). Lernen und komplexes Problemlösen. Bonn: Holos.

Beckmann, J. \& Guthke, J. (1995). Complex problem solving, intelligence, and leaming ability. In P. A. Frensch \& J. Funke (Eds.), Complex problem solving: The European perspective (pp. 177-200). Hillsdale, NJ: Erlbaum.

Berry, D. C. (1991). The role of action in implicit learning. Quarterly Journal of Experimental Psychology, 43A, $881-906$.

Berry, D. C. \& Broadbent, D. E. (1984). On the relationship between task performance and associated verbalisable knowledge. Quarterly Journal of Experimental Psychol. ogy, 36A, 209-231.

Buchner, A. (1992). Implizites Lernen: Probleme und Per. spektiven. Weinheim: Psychologie Verlags Union.

Bums, B. D. \& Vollmeyer, R. (1996). Goals and problem solving: Learning as search of three spaces. In G. W. Cottrell (Ed.), Proceedings of the Eighteenth Annual Conference of the Cognitive Science Socien (pp. 23-24). Hillsdale, NJ: Erlbaum.

Cervone, D., Jiwani, N. \& Wood, R. (1991). Goal setting and the differential influence of self-regulatory processes on complex decision-making performance. Journal of Per. sonaling and Social Psychology, 61, 257-266.

Dömer, D. (1987). Problemlösen als Informationsverarbeitung (3. Auf.). Stuttgart: Kohlhammer.

Dömer, D., Kreuzig, H. W., Reither, F. \& Stäudel, T. (Hrsg.). (1983). Lohllausen. Vom Umgang mit Unbestimmtheit und Komplexität. Bern: Huber.

Earley, P. C., Connolly, T. \& Ekegren, G. (1989). Goals, strategy development, and task performance: Some limits on the efficacy of goal setting. Journal of Applied Psychologi; 74, 24-33.

Fahrenberg. J., Hampel, R. \& Selg, H. (1984). Das Freiburger Persönlichkeitsinventar (FPl) (4. Aufl.). Göttingen: Hogrefe.

Funke. J. (1991). Solving complex problems: Exploration and control of complex systems. In R. J. Stemberg \& P. A. Frensch (Eds.). Complex problem solving: Principles and mechanisms (pp. 185-222). Hillsdale, NJ: Erlbaum. 
Funke, J. (1995). Experimental research on complex problem solving. In P. A. Frensch \& J. Funke (Eds.). Complex problem solving: The European perspective (pp. 243-268). Hillsdale. NJ: Erlbaum.

Funke, J. \& Müller, H. (1988). Eingreifen und Prognostizieren als Determinanten von Systemidentifikation und Systemsteuerung. Spraclie \& Kognition, 7. 176-186.

Geddes, B. W. \& Stevenson, R. J. (1997). Explicit leaming of a dynamic system with a non-salient pattem. Quarierly. Jourial of Experimenial Psychology, 50A. 742-765.

Gordon. D. \& Subramanian, D. (1997). A cognitive model of learning to navigate. In M. G. Shafto \& P. Langley (Eds.), Proceedings of the Nineteenth Annual Conference of the Cognitive Science Society (pp. 271-276). Hillsdale. NJ: Erlbaum.

Kanfer, R. \& Ackerman. P. L. (1989). Motivation and cognitive abilities: An integrative/aptitude-treatment interaction approach to skill acquisition. Journal of Applied Psychology; 74, 657-690.

Klahr, D. \& Dunbar, K. (1988). Dual space search during scientific reasoning. Cognitive Science, 12, 1-55.

Newell, A. \& Simon, H. A. (1972). Human problem solving. Englewood Cliffs, NJ: Prentice Hall.

Nhouyvanisvong, A. \& Koedinger, K. R. (1998). Goal specificity and learning: Reinterpretation of the data and cognitive theory. In M. A. Gernsbacher \& S. J. Derty (Eds.), Proceedings of the Twentieth Annual Conference of the Cognitive Science Sociery (pp. 764-769). Hillsdale, NJ: Erlbaum.

PreuBler, W. (1996). Zur Rolle expliziten Wissens bei der Steuerung dynamischer Systeme. Zeitschrift für Experimentelle Psychologie, 43, 399-434.

PreuBler, W. (1997). Effekte des Kontexts auf den Wissenserwerb bei der Steuenung eines dynamischen Systems. Sprache \& Kognition, 16, 48-59.

PreuBler, W. (1998). Strukturwissen als Voraussetzung für die Steuerung komplexer dynamischer Systeme. Zeitschrift für Experimentelle Psychologie, 45, 218-240.

Putz-Osterloh, W. (1993). Strategies for knowledge acquisition and transfer of knowledge in dynamic tasks. In G. Strube \& K. F. Wender (Eds.), The cognitive psychology of knowledge (pp. 331-350). Amsterdam: Elsevier.

Riefer, D. M. \& Batchelder, W. H. (1988). Multinomial modeling and the measurement of cognitive processes. Psychological Review, 95, 318-339.

Schauble, L., Glaser, R., Raghavan, K. \& Reiner, M. (1992). The integration of knowledge and experimentation strategies in understanding a physical system. Applied Cognitive Psychology: 6, 321-343.

Schoppek, W. (1997). Wissen bei der Steuenung dynamischer Systeme - ein prozeßorientierter Forschungsansatz. Zeitschrift fiur Psychologie, 205, 269-295.

Schoppek, W. (1998). Toriard a theon of the control of dynamic systerns. Paper presented at the second European Conference on Cognitive Modeling in Nottingham, UK.

Schwarzer, R. \& Jenusalem, M. (1981). Fragebogen zur Selbstnirksamkeitsenwartung. Unveröffentlichtes Manuskript.

Seger, C. A. (1994). Implicit learning. Psychological Bulletin, IIS, 163-196.

Simon, H. A. \& Lea, G. (1974). Problem solving and rule induction: A unified view. In L. W. Gregg (Ed.), Knowledge and cognition (pp. 105-127). Hillsdale. NJ: Erlbaum.
Süß. H.-M. (1996). Intelligenz, Wissen und Problemlösen. Göllingen: Hogrefe.

Stevenson, R. J., Geddes, B. W., Sumner. J. B. \& Travis. B. M. K. (1998). Look and learn: Observational leaming of rules and instances. In M. A. Gernsbacher \& S. J. Derry (Eds.), Proceedings of the Twentieth Annual Conference of the Cognitive Science Socien. (pp. 1025-1030). Hillsdale, NJ: Erlbaum.

Tschirgi, J. E. (1980). Sensible reasoning: A hypothesis about hypotheses. Child Development, 5I, 1-10.

Tubbs, M. E. (1986). Goal setting: A meta-analytic examination of the empirical evidence. Journal of Applied Psychology. $7 I, 474-483$

VanLehn, K. (1989). Problem solving and cognitive skill. In M. I. Posner (Ed.), Foundations of cognitive science (pp. 527-579). Cambridge, MA: MIT Press.

Vollmeyer, R. \& Burns, B. D. (1996). Hypotheseninstruktion und Zielspezifität: Bedingungen, die das Erlemen und Kontrollieren eines komplexen Systems beeinflussen. Zeitschrift fïr Experimentelle Psychologie, 43, 657-683.

Vollmeyer, R., Burns, B. D. \& Holyoak. K. J. (1996). The impact of goal on strategy use and the acquisition of problem structure. Cognirive Science, 20, 75-100.

Vollmeyer, R. \& Rheinberg, F. (1998). Motivationale Einflüsse auf Erwerb und Anwendung von Wissen in einem computersimulierten System. Zeitschrifi für Pädagogische Psychologie, 12, 11-23.

Vollmeyer, R. \& Rheinberg, F. (in press). Motivation and metacognition when learning a complex system. European Journal of Psychology of Education.

Vollmeyer, R., Rollett, W. \& Rheinberg, F. (1997). How motivation affects learning. In M. G. Shafto \& P. Langley (Eds.), Proceedings of the Nineteenth Annual Conference of the Cognitive Science Sociery (pp. 796-801). Hillsdale, NJ: Erlbaum.

Wallach, D. (1998). Komplexe Regelungsprozesse. Eine kognitionswissenschaftliche Analyse. Wiesbaden: Deutscher Universitäts-Verlag.

Weinert, F. E. (1984). Metakognition und Motivation als Deteminanten der Lernaktivităt: Einfühnung und Überblick. In F. E. Weinert \& R. H. Kluwe (Hrsg.), Merakognition, Motivation und Lernen (S. 9-21). Stuttgart: Kohlhammer.

Wood, R. E. \& Bandura, A. (1989). Impact of conceptions of ability on self-regulatory mechanisms and complex decision making. Journal of Personality and Social Psychology, 56, 407-415.

Wood, R. E., Bandura, A. \& Bailey, T. (1990). Mechanisms governing organizational performance in complex decisionmaking environments. Organizational Behavior and Human Decision Processes, 46, 181-201.

\section{Dr. Regina Vollmeyer}

Institut für Psychologie der Universität Potsdam

Postfach 601553

D-14415 Potsdam

E-Mail: vollmeye@rz.uni-potsdam.de

Prof. Dr. Joachim Funke

Psychologisches Institut der Ruprecht-Karls-Universität Heidelberg

Hauptstr. 47-51, D-69117 Heidelberg

E-Mail: joachim.funke@urz.uni-heidelberg.de 